Tailored coupled cluster singles and doubles method applied to calculations on molecular structure and harmonic vibrational frequencies of ozone

Osamu Hino, Tomoko Kinoshita, and Garnet Kin-Lic ChanRodney J. Bartlett

Citation: J. Chem. Phys. 124, 114311 (2006); doi: 10.1063/1.2180775

View online: http://dx.doi.org/10.1063/1.2180775

View Table of Contents: http://aip.scitation.org/toc/jcp/124/11

Published by the American Institute of Physics 


\title{
Tailored coupled cluster singles and doubles method applied to calculations on molecular structure and harmonic vibrational frequencies of ozone
}

\author{
Osamu Hino, ${ }^{\text {a) }}$ Tomoko Kinoshita, and Garnet Kin-Lic Chan \\ Department of Chemistry and Chemical Biology, Cornell University, Ithaca, New York 14853-1301 \\ Rodney J. Bartlett \\ Quantum Theory Project, University of Florida, Gainesville, Florida 32611
}

(Received 16 November 2005; accepted 3 February 2006; published online 20 March 2006)

\begin{abstract}
To assess the separation of dynamic and nondynamic correlations and orbital choice, we calculate the molecular structure and harmonic vibrational frequencies of ozone with the recently developed tailored coupled cluster singles and doubles method (TCCSD). We employ the Hartree-Fock and complete active space (CAS) self-consistent field (SCF) orbitals to perform TCCSD calculations. When using the Hartree-Fock orbitals, it is difficult to reproduce the experimental vibrational frequency of the asymmetric stretching mode. On the other hand, the TCCSD based on the CASSCF orbitals in a correlation consistent polarized valence triple zeta basis yields excellent results with the two symmetric vibrations differing from the experimental harmonic values by $2 \mathrm{~cm}^{-1}$ and the asymmetric vibration differing by $9 \mathrm{~cm}^{-1}$. (C) 2006 American Institute of Physics.
\end{abstract}

[DOI: $10.1063 / 1.2180775]$

\section{INTRODUCTION}

The molecular structure and harmonic vibrational frequencies of ozone have served as prototypical examples of the role of nondynamic correlation in a molecule where no bond breaking is involved. Because the electronic structure of ozone has a multiconfigurational nature ${ }^{1-3}$ and is quite sensitive to geometrical distortion, it has been recognized to be a demanding test to calculate its equilibrium molecular structure and vibrational frequencies for all quantum chemical methods. Earlier works are mostly based on the configuration interaction (CI) approach. ${ }^{1-6}$ Hay et al. made pioneering contributions to the study of the electronic structure of ozone. ${ }^{1,2}$ Considering the orbital issue, Adler-Golden et al. ${ }^{4}$ reported complete active space self-consistent field (CASSCF) results on the potential energy surface and spectroscopic constants of ozone. They employed a CAS in which all $2 p$ orbitals in three oxygen atoms are active [12 electrons in 9 spatial orbitals, CAS $(12,9)]$. The CASSCF vibrational frequencies were underestimated in comparison with experiment ${ }^{5,6}$ while they were qualitatively correct in terms of the order of frequencies. Yamaguchi et al. ${ }^{7}$ showed that the two-configuration SCF (TCSCF) method performed much better than the Hartree Fock for the molecular structure, but failed to give a correct ordering of the symmetric stretching mode, $\omega_{1}$, and asymmetric stretching one, $\omega_{3}$. When generalized to TCSCF-CI to introduce some dynamic correlation, the incorrect order of the two stretching modes remained. ${ }^{8}$ Peterson et al. ${ }^{9}$ performed larger CASSCF calculation with the full valence CAS [18 electrons in 12 spatial orbitals, CAS $(18,12)]$. The calculated molecular structure was similar while the vibrational frequencies were improved

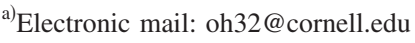

compared with Ref. 4. Borowski et al. ${ }^{10}$ attempted to incorporate dynamic correlation with the second order CASSCF perturbation theory ${ }^{11,12}$ (CASPT2) and the multireference (MR) CI with selected reference functions extracted from restricted active space (RAS) SCF (Ref. 13) calculations. The CASPT2 results were more or less the same as those of the CASSCF. The most successful results were obtained from a ten reference MRCI with a $4 s 3 p 2 d$ atomic natural orbital $^{14}$ (ANO) basis. Through these studies it appears to be indispensable to handle the multiconfigurational character of the electronic structure properly while also incorporating dynamic correlation to obtain accurate properties of ozone.

In the mean time, other groups attacked this problem with single reference many-body methods, many-body perturbation theory ${ }^{15,16}$ (MBPT) and coupled cluster ${ }^{15-19}$ (CC) theory. It is now well known that such methods typically offer superior convergence to the full CI energies, ${ }^{20}$ so the question was whether the convergence to the full $\mathrm{CI}$ is fast enough to offset the lack of explicit nondynamic correlation occurring in ozone. It was shown that the MBPT methods performed reasonably well for the two symmetric modes, but MBPT(2) and MBPT(4) were worse than the Hartree-Fock result for $\omega_{3}{ }^{15,16}$ However, the iterative CC singles and doubles $^{21}$ (CCSD) method reproduced the correct order of the vibrational frequencies ${ }^{15-19}$ and outperformed the multireference TCSCF-CISD. However, when trying to add a triple correction to CCSD, the CCSD $[\mathrm{T}]$ failed badly, making $\omega_{3}$ imaginary. ${ }^{15,16}$ This problem was dramatically improved $^{18,19,22}$ by adding the fifth order correction, $E_{S T}^{[5]}$, to the CCSD or quadratic CISD ${ }^{23}$ (QCISD) energy, namely, the $\operatorname{CCSD}(\mathrm{T})\left(\right.$ Refs. 24 and 25) or QCISD(T). ${ }^{23}$ The calculated structure and vibrational frequencies of the $\operatorname{CCSD}(\mathrm{T})$ agreed with the experiment in a double zeta polarization ${ }^{26}$ (DZP) 
basis. $^{18,19}$ However, larger basis sets tend to hurt the agreement. $^{20,27,28}$ Using Brueckner orbitals ${ }^{29}$ with a triples correction, $\mathrm{BD}(\mathrm{T}){ }^{30}$ there is also good agreement as this differs from $\operatorname{CCSD}(\mathrm{T})$ only by an additional rotation of the orbitals to make $T_{1}=0$.

The role of higher excitations in many-body methods has been considered. Watts and Bartlett performed CCSDT calculations. ${ }^{27}$ CCSDT (Refs. 31 and 32) with the correlation consistent polarized valence triple zeta (cc-pVTZ) basis provided a correct description of the properties of ozone. $\mathrm{Ku}$ charski and Bartlett ${ }^{28}$ augmented CCSDT with their factorized quadruples correction, $\operatorname{CCSDT}\left(Q_{f}\right),{ }^{34}$ which further improved the results from the CCSDT.

Other used extensions of MR-CI. Leininger and Schaefer III $^{35}$ applied the CISD[TQ] (Ref. 36) method, which is a kind of MRCISD with reduced number of references, to calculate the properties of ozone. Contrary to several previous CI based studies, the CISD[TQ] yielded the correct order of vibrational frequencies. Xie et al. $^{37}$ surveyed the potential energy surface of ozone with the internally contracted (ic) MRCI (Refs. 38 and 39) plus the Davidson correction, 40 icMRCI $+Q$. They performed CASSCF with the CAS $(12,9)$ prior to icMRCI and incorporated dynamic correlation with the icMRCI $+Q$. They employed the correlation consistent polarized valence quadruple zeta ${ }^{33}$ (cc-pVQZ) basis supplemented with diffuse functions, cc-pVQZ+ $(1 s 1 p) .{ }^{37}$ The molecular structure and vibrational frequencies were in satisfactory agreement with experiment.

Multireference many-body methods were also applied. Hubac et al. $^{41}$ applied the state-specific MR BrillouinWigner (BW) CCSD method. ${ }^{42}$ Their calculations failed in giving accurate predictions on $\omega_{3}$, and it was explained by the fact that they adopted only two references for the MR BWCCSD calculations as suggested by the observed failure of the TCSCF-CISD. Li and Paldus ${ }^{43}$ performed reduced multireference (RMR) CCSD (Ref. 44) calculations for the properties of ozone. The two reference RMR CCSD had a similar behavior as the TCSCF-CISD and two reference MR BWCCSD. They also performed the more appropriate three reference RMR CCSD, which provided the correct order of the vibrational frequencies. We should also mention the MR averaged quadratic CC (MR-AQCC) results of Szalay and Bartlett $^{45}$ which though less rigorous than other MR-CC methods has the notable advantage that analytical gradients are available, making the application to more general molecules routine.

Other generalizations of operationally single reference CC (SR-CC) have been considered. Sattelmeyer et al. developed the $2 h$ and $3 h$ - $p$-type coupled-cluster-based TammDancoff approximation (EOMDIP) method and tested its performance. ${ }^{46}$ Their calculation reconfirmed that it is still difficult to estimate $\omega_{3}$ accurately. The locally renormalized CCSD(T) [LR-CCSD(T)] methods were also applied to calculate the properties of ozone. ${ }^{47}$ Among a number of different corrections to the CCSD, the IIIB correction gave the closest results to the experiment.

Recently, we have proposed the tailored CC (TCC) method, ${ }^{48}$ which tailors the CC method in combination with the CI so that we can manipulate electronic states with quasidegenerate character within a framework of the SR-CC method. Ozone's vibrational frequencies offer a critical test for the method. In Sec. II, we briefly review the TCCSD method. In Sec. III, we show numerical results for the properties of ozone. We compare the TCCSD results with those obtained by other theories. In Sec. IV, we offer some conclusions.

\section{TAILORED CCSD METHOD}

In the tailored $\mathrm{CC}$ method, the $\mathrm{CC}$ amplitude is chosen to be explicitly divided into a dynamic and a nondynamic part as

$$
\left|\Psi_{\mathrm{TCC}}\right\rangle=\exp (\hat{T})\left|\Psi_{0}\right\rangle=\exp \left(\hat{T}^{\mathrm{ext}}+\hat{T}^{\mathrm{CAS}}\right)\left|\Psi_{0}\right\rangle,
$$

where $\Psi_{0}$ is the reference determinant, the amplitude $\hat{T}^{\mathrm{CAS}}$ is extracted from the CI coefficients of the CAS-CI wave function (if we employ CASSCF orbitals, this becomes a CASSCF wave function) so that it covers nondynamic correlation effect, and the external amplitude $\hat{T}^{\text {ext }}$ is determined by the restricted CC procedure in which $\hat{T}^{\text {CAS }}$ is frozen. In this study, we use the following TCCSD ansatz:

$$
\begin{aligned}
\left|\Psi_{\mathrm{TCCSD}}\right\rangle & =\exp \left(\hat{T}_{1}^{\mathrm{ext}}+T_{2}^{\mathrm{ext}}\right) \exp \left(\hat{T}_{1}^{\mathrm{CAS}}+\hat{T}_{2}^{\mathrm{CAS}}\right)\left|\Psi_{0}\right\rangle \\
& \simeq \exp \left(\hat{T}_{1}^{\mathrm{ext}}+\hat{T}_{2}^{\mathrm{ext}}\right)\left|\Psi_{\mathrm{CAS}-\mathrm{CI}}\right\rangle .
\end{aligned}
$$

The amplitudes of the cluster operators, $\hat{T}_{1}^{\mathrm{CAS}}$ and $\hat{T}_{2}^{\mathrm{CAS}}$, are extracted from the CAS-CI wave function,

$$
\left|\Psi_{\mathrm{CAS}-\mathrm{CI}}\right\rangle=\left(1+\hat{C}_{1}+\hat{C}_{2}+\hat{C}_{3}+\cdots+\hat{C}_{n}\right)\left|\Psi_{0}\right\rangle,
$$

where $\hat{C}_{1}, \hat{C}_{2}, \ldots, \hat{C}_{n}$ are the $n$th order CI expansion operators. Variants of this idea have been considered by $\mathrm{Li}$ and Paldus and Paldus and $\mathrm{Li}^{44}$ and Oliphant and Adamowicz, ${ }^{49}$ but unlike those authors, we insist upon an inexpensive CCSD approximation. This means all higher-excitation nondynamic correlation effects are subsumed into just $\hat{T}_{1}^{\text {CAS }}$ and $\hat{T}_{2}^{\text {CAS }}$. Furthermore, these contributions are effectively decoupled from higher excitations as discussed elsewhere. ${ }^{48}$ The CCSD approximation enables the rapid calculation of electron correlation compared with adding $T_{3}, T_{4}$, etc., with CC theory, or doing genuine multireference $\mathrm{CC}$ calculations.

From the well-known relationship between the $\mathrm{CC}$ and CI expansions, $\hat{T}_{1}^{\mathrm{CAS}}$ and $\hat{T}_{2}^{\mathrm{CAS}}$ are written as

$$
\begin{aligned}
& \hat{T}_{1}^{\mathrm{CAS}}=\hat{C}_{1}, \\
& \hat{T}_{2}^{\mathrm{CAS}}=\hat{C}_{2}-\frac{1}{2}\left[\hat{C}_{1}\right]^{2} .
\end{aligned}
$$

Straightforwardly, those amplitudes are obtained as

$$
\begin{aligned}
& t_{i}^{a}=c_{i}^{a}, \quad a, i \in \mathrm{CAS}, \\
& t_{i j}^{a b}=c_{i j}^{a b}-\left(c_{i}^{a} c_{j}^{b}-c_{j}^{a} c_{i}^{b}\right), \quad a, b, i, j \in \mathrm{CAS},
\end{aligned}
$$

where $a, b, \ldots$ and $i, j, \ldots$ indicate unoccupied and occupied orbitals with respect to the formal reference determinant $\Psi_{0}$.

The external cluster operators, $\hat{T}_{1}^{\text {ext }}$ and $\hat{T}_{2}^{\text {ext }}$, are defined as 
TABLE I. CASSCF calculations on the equilibrium geometries and vibrational frequencies of the ground state of ozone with the cc-pVDZ basis. The numbers in parentheses show the relative errors (\%) from the experimental values. The units are $\AA$ for the bond length $R_{\mathrm{O}-\mathrm{O}},{ }^{\circ}$ for the bond angle $\theta$, and $\mathrm{cm}^{-1}$ for vibrational frequencies.

\begin{tabular}{lccccc}
\hline \hline Level & $R_{\mathrm{O}-\mathrm{O}}$ & $\theta$ & $\omega_{1}\left(a_{1}\right)$ & $\omega_{2}\left(a_{1}\right)$ & $\omega_{3}\left(b_{2}\right)$ \\
\hline Hartree-Fock & 1.197 & 119.0 & 1533 & 867 & 1423 \\
& $(5.90)$ & $(1.88)$ & $(35.1)$ & $(21.1)$ & $(30.7)$ \\
CAS $(2,2)^{\mathrm{a}}$ & 1.249 & 115.3 & 1160 & 794 & 1460 \\
& $(1.81)$ & $(1.28)$ & $(2.20)$ & $(10.9)$ & $(34.1)$ \\
CAS $(6,5)^{\mathrm{b}}$ & 1.252 & 117.3 & 1170 & 764 & 1695 \\
& $(1.57)$ & $(0.43)$ & $(3.08)$ & $(6.70)$ & $(55.6)$ \\
CAS $(8,7)^{\mathrm{c}}$ & 1.290 & 114.8 & 1039 & 707 & 1193 \\
& $(1.42)$ & $(1.71)$ & $(8.46)$ & $(1.26)$ & $(9.55)$ \\
CAS $(10,8)^{\mathrm{d}}$ & 1.289 & 115.3 & 1049 & 706 & 1274 \\
& $(1.34)$ & $(1.28)$ & $(7.58)$ & $(1.40)$ & $(17.0)$ \\
CAS $(12,9)^{\mathrm{e}}$ & 1.290 & 116.4 & 1059 & 696 & 964 \\
& $(1.42)$ & $(0.34)$ & $(6.70)$ & $(2.79)$ & $(11.5)$ \\
Experiment & 1.272 & 116.8 & 1135 & 716 & 1089 \\
\hline \hline
\end{tabular}

${ }^{\mathrm{a}} \mathrm{CAS}$ with 2 electrons in spatial orbitals, $\left[1 a_{2} 2 b_{1}\right]$.

${ }^{\mathrm{b}} \mathrm{CAS}$ with 6 electrons in spatial orbitals, $\left[5 a_{1} 6 a_{1} 1 a_{2} 2 b_{1} 5 b_{2}\right]$.

${ }^{\mathrm{c}} \mathrm{CAS}$ with 8 electrons in spatial orbitals, [5a $\left.4 a_{2} 6 a_{1} 1 a_{2} 2 b_{1} 7 a_{1} 5 b_{2}\right]$.

${ }^{\mathrm{d}}$ CAS with 10 electrons in spatial orbitals, [ $\left.5 a_{1} 1 b_{1} 4 b_{2} 6 a_{1} 1 a_{2} 2 b_{1} 7 a_{1} 5 b_{2}\right]$.

${ }^{\mathrm{e}} \mathrm{CAS}$ with 12 electrons in spatial orbitals, $\left[5 a_{1} 3 b_{2} 1 b_{1} 4 b_{2} 6 a_{1} 1 a_{2} 2 b_{1} 7 a_{1} 5 b_{2}\right]$.

$$
\begin{aligned}
& \hat{T}_{1}^{\mathrm{ext}}=\sum_{a i} t_{i}^{a} a^{\dagger} i, \quad\{a, i\} \mp \mathrm{CAS}, \\
& \hat{T}_{2}^{\mathrm{ext}}=\frac{1}{4} \sum_{a b i j} t_{i j}^{a b} a^{\dagger} b^{\dagger} j i, \quad\{a, b, i, j\} \mp \mathrm{CAS} .
\end{aligned}
$$

The amplitudes of the external cluster operators are determined to satisfy the following equations:

$$
\begin{aligned}
& \left\langle\Psi_{i}^{a}\left|H \exp \left(\hat{T}_{1}^{\mathrm{ext}}+\hat{T}_{2}^{\mathrm{ext}}\right) \exp \left(\hat{T}_{1}^{\mathrm{CAS}}+\hat{T}_{2}^{\mathrm{CAS}}\right)\right| \Psi_{0}\right\rangle_{c}=0, \\
& \{a, i\} \mp \mathrm{CAS}, \\
& \left\langle\Psi_{i j}^{a b}\left|H \exp \left(\hat{T}_{1}^{\mathrm{ext}}+\hat{T}_{2}^{\mathrm{ext}}\right) \exp \left(\hat{T}_{1}^{\mathrm{CAS}}+\hat{T}_{2}^{\mathrm{CAS}}\right)\right| \Psi_{0}\right\rangle_{c}=0, \\
& \quad\{a, b, i, j\} \leftarrow \mathrm{CAS},
\end{aligned}
$$

where the subscript $c$ means that we only consider the connected elements. The total electronic energy is given by

$$
E=\left\langle\Psi_{0}\left|H \exp \left(\hat{T}_{1}^{\mathrm{ext}}+\hat{T}_{2}^{\mathrm{ext}}\right) \exp \left(\hat{T}_{1}^{\mathrm{CAS}}+\hat{T}_{2}^{\mathrm{CAS}}\right)\right| \Psi_{0}\right\rangle .
$$

A more detailed description can be found in the previous paper. ${ }^{48}$

\section{RESULTS AND DISCUSSION}

We perform two types of TCCSD calculations. One is based on the restricted Hartree-Fock (RHF) orbitals and the other is based on the CASSCF orbitals. CASSCF orbitals are taken from the program package GAMESS. ${ }^{50}$ These TCCSD calculations employ the CAS $(12,9)$ where $2 p$ orbitals of each oxygen atom are active (12 electrons in 9 spatial orbitals, $\left.\left[5 a_{1} 1 b_{1} 3 b_{2} 4 b_{2} 6 a_{1} 1 a_{2} 2 b_{1} 7 a_{1} 5 b_{2}\right]\right)$ as in Ref. 4 . As explained below, this is because we would not obtain a qualitatively correct equilibrium geometry and harmonic vibrational frequencies of ozone by using a smaller CAS than the $\operatorname{CAS}(12,9)$.
Within a simple picture, the electronic ground state of ozone can be viewed as a two-determinant state composed of the configurations [(core) $\left.4 b_{2}^{2} 6 a_{1}^{2} 1 a_{2}^{2}\right]$ and [(core) $\left.4 b_{2}^{2} 6 a_{1}^{2} 2 b_{1}^{2}\right] .{ }^{1-3}$ However, earlier studies show that one would get qualitatively incorrect results with the TCSCF. ${ }^{7}$ This is equivalent to a CASSCF, CAS(2,2), which contains 2 electrons in 2 spatial orbitals, $\left[1 a_{2} 2 b_{1}\right]$. In addition, incorporating the dynamic correlation with the MRCISD or Brillouin-Wigner MRCC is not enough to recover accurate results. ${ }^{8,41}$ Therefore, before performing TCCSD calculations, we tried several CASSCF calculations for the molecular structure and vibrational frequencies of ozone to identify the smallest CAS which can maintain reasonably accurate results.

To perform the above task, we use the information from the stability analysis of the RHF wave function. When we use the correlation consistent polarized valence double zeta (cc-pVDZ) and the cc-pVTZ basis, there is an instability, which corresponds to the electron excitation $1 a_{2} \rightarrow 2 b_{1}$, and this suggests the CAS $(2,2)$. However, there are several electron excitations which have very small positive eigenvalues, suggesting that it is necessary to cover these excitations within the CAS in order to obtain a qualitatively correct SCF wave function. The electron excitation with the smallest positive eigenvalue is basically described as the linear combination of excitations $5 a_{1} \rightarrow 2 b_{1}, 6 a_{1} \rightarrow 2 b_{1}$, and $1 a_{2} \rightarrow 5 b_{2}$. This recommends a $\operatorname{CAS}(6,5)$ which contains 6 electrons in 5 spatial orbitals, $\left[5 a_{1} 6 a_{1} 1 a_{2} 2 b_{1} 5 b_{2}\right]$. We keep extending the CAS in the same way until we reach satisfactory results. These procedures are summarized in Table I. Table I only shows results with the cc-pVDZ basis because we see similar tendencies in the results with the cc-pVTZ basis. According to Table I, we conclude that the $\operatorname{CAS}(12,9)$ is the smallest CAS that gives a correct description of the electronic ground state of ozone because we cannot obtain a correct order of the frequencies by using smaller CASs. One might be able to 
TABLE II. Equilibrium geometries and vibrational frequencies of the ground state of ozone with the cc-pVDZ basis. In the correlated calculations, all electrons are correlated. The numbers in parentheses show the relative errors (\%) from the experimental values. The units are Å for the bond length $R_{\mathrm{O}-\mathrm{O}},{ }^{\circ}$ for the bond angle $\theta$, and $\mathrm{cm}^{-1}$ for vibrational frequencies.

\begin{tabular}{|c|c|c|c|c|c|}
\hline Theory & $R_{\mathrm{O}-\mathrm{O}}$ & $\theta$ & $\omega_{1}\left(a_{1}\right)$ & $\omega_{2}\left(a_{1}\right)$ & $\omega_{3}\left(b_{2}\right)$ \\
\hline Hartree-Fock & $\begin{array}{l}1.197 \\
(5.90)\end{array}$ & $\begin{array}{l}119.0 \\
(1.88)\end{array}$ & $\begin{array}{c}1533 \\
(35.1)\end{array}$ & $\begin{array}{c}867 \\
(21.1)\end{array}$ & $\begin{array}{c}1423 \\
(30.7)\end{array}$ \\
\hline CAS-CI & $\begin{array}{l}1.318 \\
(3.62)\end{array}$ & $\begin{array}{l}116.5 \\
(0.27)\end{array}$ & $\begin{array}{c}945 \\
(16.7)\end{array}$ & $\begin{array}{c}646 \\
(9.78)\end{array}$ & $\begin{array}{c}1158 \\
(6.34)\end{array}$ \\
\hline CASSCF & $\begin{array}{l}1.290 \\
(1.42)\end{array}$ & $\begin{array}{l}116.4 \\
(0.34)\end{array}$ & $\begin{array}{c}1059 \\
(6.70)\end{array}$ & $\begin{array}{c}696 \\
(2.79)\end{array}$ & $\begin{array}{c}964 \\
(11.5)\end{array}$ \\
\hline TCCSD/CAS-CI & $\begin{array}{l}1.289 \\
(1.34)\end{array}$ & $\begin{array}{l}116.3 \\
(0.43)\end{array}$ & $\begin{array}{c}1100 \\
(3.08)\end{array}$ & $\begin{array}{c}699 \\
(2.37)\end{array}$ & $\begin{array}{c}1282 \\
(17.7)\end{array}$ \\
\hline TCCSD/CASSCF & $\begin{array}{l}1.289 \\
(1.34)\end{array}$ & $\begin{array}{l}116.3 \\
(0.43)\end{array}$ & $\begin{array}{c}1089 \\
(4.05)\end{array}$ & $\begin{array}{c}697 \\
(2.65)\end{array}$ & $\begin{array}{r}1030 \\
(5.42)\end{array}$ \\
\hline CCSD & $\begin{array}{l}1.258 \\
(1.10)\end{array}$ & $\begin{array}{l}117.3 \\
(0.43)\end{array}$ & $\begin{array}{c}1252 \\
(10.3)\end{array}$ & $\begin{array}{c}754 \\
(5.31)\end{array}$ & $\begin{array}{c}1238 \\
(13.7)\end{array}$ \\
\hline CCSD/CASSCF & $\begin{array}{l}1.257 \\
(1.19)\end{array}$ & $\begin{array}{l}117.1 \\
(0.26)\end{array}$ & $\begin{array}{c}1255 \\
(10.6)\end{array}$ & $\begin{array}{c}761 \\
(5.99)\end{array}$ & $\begin{array}{c}1298 \\
(19.2)\end{array}$ \\
\hline $\operatorname{CCSD}(\mathrm{T})$ & $\begin{array}{l}1.284 \\
(0.94)\end{array}$ & $\begin{array}{l}116.7 \\
(0.09)\end{array}$ & $\begin{array}{c}1118 \\
(1.50)\end{array}$ & $\begin{array}{c}705 \\
(1.54)\end{array}$ & $\begin{array}{c}978 \\
(10.2)\end{array}$ \\
\hline $\operatorname{CCSD}(\mathrm{T}) / \mathrm{CASSCF}$ & $\begin{array}{l}1.288 \\
(1.26)\end{array}$ & $\begin{array}{l}116.7 \\
(0.09)\end{array}$ & $\begin{array}{c}1095 \\
(3.52)\end{array}$ & $\begin{array}{c}695 \\
(3.20)\end{array}$ & $\begin{array}{c}1050 \\
(3.58)\end{array}$ \\
\hline Experiment & 1.272 & 116.8 & 1135 & 716 & 1089 \\
\hline
\end{tabular}

reduce the number of configurations for the SCF calculation by careful choice of important ones, but that procedure could be complicated.

In addition to the TCCSD calculations, we calculate the equilibrium geometry and vibrational frequencies of ozone with Hartree Fock, CAS-CI (using Hartree-Fock orbitals), CASSCF, CCSD, and CCSD(T) for comparison. In Table II we show the results with the cc-pVDZ basis. The HartreeFock method predicts a 5.90\% smaller bond length, 1.88\% larger bond angle, and considerably overestimates the vibrational frequencies as compared with the experimental values. These are improved with the CAS-CI. However, the bond length is calculated to be $3.62 \%$ larger, the two $a_{1}$ frequencies, $\omega_{1}$ and $\omega_{2}$, are $13.2 \%$ underestimated on average, and the $b_{2}$ frequency, $\omega_{3}$, is $6.34 \%$ overestimated. We can add dynamic correlation to the CAS-CI wave function with the TCCSD [TCCSD/CAS-CI]. Except for $\omega_{3}$, the TCCSD/ CAS-CI improves the accuracy of the calculated results. In this case, the bond length, $\omega_{1}$, and $\omega_{2}$ become closer to those of experiment while the bond angle is estimated to be $0.2^{\circ}$ less than that obtained from CAS-CI and $\omega_{3}$ is overestimated by $17.7 \%$. It is interesting to compare results obtained with CAS-CI based TCCSD with those of the standard CCSD. The quality of the molecular geometry is more or less the same. $\omega_{1}$ and $\omega_{2}$ with the TCCSD/CAS-CI are more accurate than those with CCSD while the $13.7 \%$ error in the CCSD calculation of $\omega_{3}$ is smaller than that of the TCCSD/CAS-CI. At this level of basis set, it is hard to tell which is more reliable to calculate equilibrium geometry and vibrational frequencies of ozone. If we can capture the quasidegenerate nature of the electronic structure of ozone with TCCSD/ CAS-CI, however, we should be able to obtain more accurate results than with the CCSD.

In this context, it is important to see what happens when we perform TCCSD using CASSCF orbitals. First, we ad- dress the CASSCF results. The CASSCF improves the bond length and bond angles $\omega_{1}$ and $\omega_{2}$ as compared with the CAS-CI results. Though $\omega_{3}$ may be overcorrected, as mentioned previously, CASSCF provides a correct order of frequencies. Incorporating dynamic correlation to the CASSCF calculations with TCCSD, all vibrational frequencies are described quite well. The average error over three frequencies is $4.04 \%$ which is slightly smaller than that with the CCSD(T) method, $4.41 \%$, while the computed equilibrium geometry with $\operatorname{CCSD}(\mathrm{T})$ is closer to the experimental structure than that with the TCCSD/CASSCF. All of these results are compromised by the small cc-pVDZ basis, however.

One can see the results with the cc-pVTZ basis in Table III. As in the cc-pVDZ basis calculations, the Hartree-Fock results have the largest errors in all properties. Interestingly, they are not very different from those with the cc-pVDZ basis. This may indicate that the Hartree-Fock calculation is almost converged at the cc-pVTZ basis level in terms of the equilibrium geometry and vibrational frequencies. In contrast, the correlated calculations behave quite differently. The CAS-CI results are all improved as compared with those of the Hartree-Fock method. However, comparing with the cc$\mathrm{pVDZ}$ calculations, the CAS-CI results behave in an inconsistent way. With the cc-pVTZ basis, the bond length and $\omega_{2}$ and $\omega_{3}$ have larger errors $(4.17 \%, 10.6 \%$, and $21.9 \%)$ than those with the cc-pVDZ basis $(3.62 \%, 9.78 \%$, and $6.34 \%)$ while the bond angle and $\omega_{1}$ with cc-pVTZ $(0.09 \%$ and $11.9 \%)$ are better than those with cc-pVDZ $(0.27 \%$ and $16.7 \%)$. This puzzling behavior becomes clearer when the dynamic correlation is taken into account with TCCSD. Except for $\omega_{3}$, all calculated results are much improved by using a cc-pVTZ basis. The average error over the four molecular constants is $0.40 \%$ while the error is $5.92 \%$ with CCSD or $0.93 \%$ with the CCSD(T). Regarding symmetric geometrical distortions, the CAS-CI wave function behaves 
TABLE III. Equilibrium geometries and vibrational frequencies of the ground state of ozone with the cc-pVTZ basis. In the correlated calculations, all electrons are correlated. The numbers in parentheses show the relative errors (\%) from the experimental values. The units are Å for the bond length $R_{\mathrm{O}-\mathrm{O}},{ }^{\circ}$ for the bond angle $\theta$, and $\mathrm{cm}^{-1}$ for vibrational frequencies.

\begin{tabular}{lccccc}
\hline \hline Theory & $R_{\mathrm{O}-\mathrm{O}}$ & $\theta$ & $\omega_{1}\left(a_{1}\right)$ & $\omega_{2}\left(a_{1}\right)$ & $\omega_{3}\left(b_{2}\right)$ \\
\hline Hartree-Fock & 1.194 & 119.3 & 1536 & 867 & 1417 \\
& $(6.13)$ & $(2.14)$ & $(35.3)$ & $(21.1)$ & $(30.1)$ \\
CAS-CI & 1.325 & 116.7 & 1000 & 640 & 1327 \\
& $(4.17)$ & $(0.09)$ & $(11.9)$ & $(10.6)$ & $(21.9)$ \\
CASSCF & 1.282 & 116.8 & 1101 & 708 & 1041 \\
& $(0.79)$ & $(0.00)$ & $(3.00)$ & $(1.12)$ & $(4.41)$ \\
TCCSD/CAS-CI & 1.278 & 116.6 & 1142 & 714 & 1357 \\
& $(0.47)$ & $(0.17)$ & $(0.67)$ & $(0.28)$ & $(24.6)$ \\
TCCSD/CASSCF & 1.274 & 116.7 & 1137 & 718 & 1098 \\
& $(0.16)$ & $(0.09)$ & $(0.18)$ & $(0.28)$ & $(0.83)$ \\
CCSD & 1.246 & 117.6 & 1287 & 770 & 1278 \\
& $(2.04)$ & $(0.68)$ & $(13.4)$ & $(7.54)$ & $(17.4)$ \\
CCSD/CASSCF & 1.244 & 117.5 & 1293 & 775 & 1339 \\
& $(2.20)$ & $(0.60)$ & $(13.9)$ & $(7.94)$ & $(22.9)$ \\
CCSD(T) & 1.271 & 117.0 & 1163 & 723 & 1072 \\
& $(0.08)$ & $(0.17)$ & $(2.47)$ & $(0.98)$ & $(1.56)$ \\
CCSD(T)/CASSCF & 1.274 & 117.1 & 1142 & 714 & 1114 \\
& $(0.16)$ & $(0.26)$ & $(0.62)$ & $(0.28)$ & $(2.30)$ \\
Experiment & 1.272 & 116.8 & 1135 & 716 & 1089 \\
\hline \hline
\end{tabular}

favorably, but it is too "stiff" against the asymmetric distortion. As has been stated, ${ }^{15}$ this is because the mixing ratio between the two important configurations, $\left[(\right.$ core $\left.) 4 b_{2}^{2} 6 a_{1}^{2} 1 a_{2}^{2}\right]$ and $\left[(\right.$ core $\left.) 4 b_{2}^{2} 6 a_{1}^{2} 2 b_{2}^{2}\right]$, is quite sensitive to the asymmetric distortion, which is closely related to $\omega_{3}$. Even if we employ the CAS $(12,9)$ and supplement the CI calculation with the TCCSD, we are unable to overcome the problem. The error in $\omega_{3}$ of the TCCSD/CAS-CI is $24.6 \%$, the largest of all except for the Hartree-Fock result. In order to calculate $\omega_{3}$ accurately, in this example, it is indispensable to optimize the wave function and orbitals with respect to an appropriate active space. The average error over the five properties obtained with the CASSCF is $1.86 \%$, quite close to that with the $\operatorname{CCSD}(\mathrm{T}), 1.32 \%$. The TCCSD/CASSCF further improves the results of CASSCF and yields the closest results to experiment in terms of the average error, $0.30 \%$.

Next, we mention properties of ozone calculated by $\operatorname{CCSD}$ and $\operatorname{CCSD}(\mathrm{T})$ with the $\operatorname{CASSCF}(12,9)$ orbitals in order to consider the orbital effects on the CC calculation. As one can see in Tables II and III, CCSD/CASSCF (CCSD with CASSCF orbitals) yields less accurate results compared with those of TCCSD/CASSCF. CCSD/HF (CCSD with Hartree-Fock orbitals) and CCSD/CASSCF give similar molecular geometry and symmetric vibrational frequencies, $\omega_{1}$ and $\omega_{2}$, but the asymmetric stretching frequency, $\omega_{3}$, becomes worse and larger than $\omega_{1}$ when using the CASSCF orbitals. This may indicate that the formal reference determinant of the CCSD/CASSCF is not an appropriate reference to perform CCSD calculation, at least for ozone. The CASSCF wave function surely describes the electronic ground state of ozone more properly than the Hartree-Fock wave function, whereas the most dominant determinant in the CASSCF wave function itself is not as heavily weighted as in the usual single reference $\mathrm{CCSD}$ calculation. $\operatorname{CCSD}(\mathrm{T})$ results are a little puzzling. Regarding the molecular geometry,
$\operatorname{CCSD}(\mathrm{T}) / \mathrm{HF}$ and CCSD(T)/CASSCF yield almost the same results for both basis sets. Concerning the symmetric vibrational frequencies, in the case of cc-pVDZ basis, $\operatorname{CCSD}(\mathrm{T}) / \mathrm{HF}$ gives results closer to experiments than those of $\operatorname{CCSD}(\mathrm{T}) / \mathrm{CASSCF}$, but the asymmetric stretching frequency of CCSD(T)/CASSCF becomes more accurate. On the other hand, the cc-pVTZ results behave completely oppositely. Then we simply consider the cc-pVTZ results to be more reliable and interpret them in the following manner. The $(\mathrm{T})$ correction improves the CCSD/CASSCF, greatly giving more accurate symmetric vibrational frequencies than those of usual CCSD along with the use of CASSCF orbitals, which are optimized to incorporate nondynamic correlation. However, (T) is not enough to recover the inappropriateness of the formal reference determinant of the CCSD/CASSCF to yield an accurate asymmetric stretching frequency. In any case, the TCCSD/CASSCF results remain most accurate and support our treatment of nondynamic correlation. In addition, as shown in Table IV, the TCCSD/CASSCF results are most accurate among those reported in the relatively recent works.

Of course, just like in all basis set dependent calculations, the basis set error can vary along with the error due to core correlation and whether core functions are added to the basis set. For example in previous CCSD(T) (Ref. 27) studies on ozone show variations for the asymmetric vibrational frequency from 970 to 1081 with basis sets. Similarly, different iterative approximations for including triples in the cc-pVTZ basis from CCSDT-lb to CCSDT also change the values from 1031 to 1216 . Only by adding $Q_{f}$ in the $\operatorname{CCSDT}\left(Q_{f}\right)$ does the convergence of the single reference CC sequence seem to approach the correct value, but it still overshoots by $23 \mathrm{~cm}^{-1}$. Yet that is far from the current TCCSD result. Doubtless, the effect of pentuples and higher excitations might also have such a numerical effect as shown in $\mathrm{N}_{2}$ (Ref. 52) and $\mathrm{C}_{2}$ (Ref. 53) examples elsewhere. 
TABLE IV. Recently calculated properties of ozone. The numbers in parentheses show the relative errors (\%) from the experimental values. The units are $\AA$ for the bond length $R_{\mathrm{O}-\mathrm{O}},{ }^{\circ}$ for the bond angle $\theta$, and $\mathrm{cm}^{-1}$ for vibrational frequencies.

\begin{tabular}{|c|c|c|c|c|c|c|c|}
\hline Theory & Basis set & $R_{\mathrm{O}-\mathrm{O}}$ & $\theta$ & $\omega_{1}\left(a_{1}\right)$ & $\omega_{2}\left(a_{1}\right)$ & $\omega_{3}\left(b_{2}\right)$ & A.E. ${ }^{a}$ \\
\hline $\mathrm{BD}(\mathrm{T})^{\mathrm{b}}$ & $\mathrm{TZ} 2 \mathrm{P}^{\mathrm{c}}$ & $\begin{array}{l}1.280 \\
(0.63)\end{array}$ & $\begin{array}{l}116.9 \\
(0.09)\end{array}$ & $\begin{array}{l}1125 \\
(0.88)\end{array}$ & $\begin{array}{c}706 \\
(1.40)\end{array}$ & $\begin{array}{l}1095 \\
(0.55)\end{array}$ & 0.71 \\
\hline $\operatorname{CCSDT}^{\mathrm{d}}$ & cc-pVTZ & $\begin{array}{l}1.274 \\
(0.16)\end{array}$ & $\begin{array}{l}116.8 \\
(0.00)\end{array}$ & $\begin{array}{l}1163 \\
(2.47)\end{array}$ & $\begin{array}{c}717 \\
(0.14)\end{array}$ & $\begin{array}{l}1117 \\
(2.57)\end{array}$ & 1.12 \\
\hline $\operatorname{CCSDT}\left(Q_{f}\right)^{\mathrm{e}}$ & cc-pVTZ & $\begin{array}{l}1.279 \\
(0.55)\end{array}$ & $\begin{array}{l}116.8 \\
(0.00)\end{array}$ & $\begin{array}{c}1133 \\
(0.18)\end{array}$ & $\begin{array}{c}709 \\
(0.98)\end{array}$ & $\begin{array}{l}1112 \\
(2.11)\end{array}$ & 0.76 \\
\hline $\mathrm{icMRCI}+Q^{\mathrm{f}}$ & $\begin{array}{c}\text { cc-pVQZ+ } \\
(1 s, 1 p)\end{array}$ & $\begin{array}{l}1.274 \\
(0.16)\end{array}$ & $\begin{array}{l}116.9 \\
(0.09)\end{array}$ & $\begin{array}{l}1125 \\
(0.88)\end{array}$ & $\begin{array}{c}719 \\
(0.42)\end{array}$ & $\begin{array}{l}1105 \\
(1.47)\end{array}$ & 0.60 \\
\hline TR BW CCSD ${ }^{g}$ & cc-pVTZ & $\begin{array}{l}1.280 \\
(0.62)\end{array}$ & $\begin{array}{l}116.3 \\
(0.43)\end{array}$ & $\begin{array}{l}1095 \\
(3.52)\end{array}$ & $\begin{array}{c}703 \\
(1.82)\end{array}$ & $\begin{array}{c}1505 \\
(38.2)\end{array}$ & 8.92 \\
\hline 3R-RMR CCSD ${ }^{\mathrm{h}}$ & DZP & $\begin{array}{l}1.277 \\
(0.39)\end{array}$ & $\begin{array}{l}116.7 \\
(0.09)\end{array}$ & $\begin{array}{c}1187 \\
(4.58)\end{array}$ & $\begin{array}{c}727 \\
(1.54)\end{array}$ & $\begin{array}{l}1156 \\
(6.15)\end{array}$ & 2.55 \\
\hline EOMDIP-CCSDT $^{\mathrm{i}}$ & cc-pVTZ & $\begin{array}{l}1.275 \\
(0.24)\end{array}$ & $\begin{array}{l}116.1 \\
(0.60)\end{array}$ & $\begin{array}{l}1139 \\
(0.35)\end{array}$ & $\begin{array}{c}724 \\
(1.12)\end{array}$ & $\begin{array}{l}1181 \\
(8.45)\end{array}$ & 2.15 \\
\hline LR-CCSD(T), IIIB $^{\mathrm{j}}$ & DZP & $\begin{array}{l}1.286 \\
(1.10)\end{array}$ & $\begin{array}{l}116.8 \\
(0.00)\end{array}$ & $\begin{array}{l}1135 \\
(0.00)\end{array}$ & $\begin{array}{c}706 \\
(1.40)\end{array}$ & $\begin{array}{l}1058 \\
(2.85)\end{array}$ & 1.07 \\
\hline TCCSD/CASSCF & cc-pVTZ & $\begin{array}{l}1.274 \\
(0.16)\end{array}$ & $\begin{array}{l}116.7 \\
(0.09)\end{array}$ & $\begin{array}{l}1137 \\
(0.18)\end{array}$ & $\begin{array}{c}718 \\
(0.28)\end{array}$ & $\begin{array}{l}1098 \\
(0.83)\end{array}$ & 0.30 \\
\hline Experiment & & 1.272 & 116.8 & 1135 & 716 & 1089 & \\
\hline
\end{tabular}

\footnotetext{
${ }^{\mathrm{a}}$ Average error.

${ }^{\mathrm{b}}$ Reference 30 .

${ }^{\mathrm{c}}$ References 26 and 51.

${ }^{\mathrm{d}}$ Reference 27.

${ }^{\mathrm{e}}$ Reference 28.

${ }^{\mathrm{f}}$ Reference 37.

${ }^{\mathrm{g}}$ Reference 41.

${ }^{\mathrm{h}}$ Reference 43.

${ }^{\mathrm{i}}$ Reference 46.

${ }^{\mathrm{j}}$ Reference 47.
}

\section{CONCLUSION}

In this study, we apply the TCCSD method to calculate the equilibrium geometry and harmonic vibrational frequencies of ozone to investigate its performance. It is important to identify an appropriate CAS. We utilize information from the stability analysis of the RHF wave function of ozone, and conclude, like others, ${ }^{4}$ that the CAS $(12,9)$ is adequate. Usually, such a CAS reference would require a huge computational effort to perform MRCISD or MRCC calculations to incorporate the dynamic correlation. However, the TCCSD is almost as cheap as the standard CCSD unless we use a very large CAS. For ozone, CASSCF with CAS $(12,9)$ is still less expensive than CCSD(T) in both cc-pVDZ and cc-pVTZ basis sets. Hence, it offers a convenient way to introduce the dynamic correlation to various types of SCF calculations.

Using cc-pVTZ basis, we find that the TCCSD/CAS-CI significantly improves upon the equilibrium geometry and the totally symmetric vibrational frequencies, $\omega_{1}$ and $\omega_{2}$, as compared with those of the CCSD, while the frequency of the asymmetric stretch, $\omega_{3}$, deteriorates. As somewhat expected, this behavior can be explained by the fact that the Hartree-Fock orbitals are optimized only for the [(core) $\left.4 b_{2}^{2} 6 a_{1}^{2} 1 a_{2}^{2}\right]$ configuration and the other important configurations such as $\left[(\right.$ core $\left.) 4 b_{2}^{2} 6 a_{1}^{2} 2 b_{1}^{2}\right]$ are not taken into account, even if we employ the CAS $(12,9)$. However, we can solve this problem by employing the CASSCF orbitals. The most prominent feature of the TCCSD/CASSCF is that the "difficult" frequency, $\omega_{3}$, can be calculated accurately. In addition, TCCSD/CASSCF yields the most accurate results with respect to the average error over the bond length, bond angle, and vibrational frequencies among the Hartree-Fock, Hartree-Fock orbital based CAS-CI, CASSCF, TCCSD/ CAS-CI, TCCSD/CASSCF, CCSD, and CCSD(T) methods in this study. We expect that the TCCSD, especially based on the CASSCF wave function and orbitals, to be capable of providing highly accurate results over a wide range of molecular structures and vibrational frequencies.

Finally we point out some aspects of the TCCSD method. First, the TCCSD is not entirely balanced in the sense that a MR-CC would be, since that method takes excitations from all relevant reference functions. Instead, the TCCSD intentionally separates the correlation problem into dynamic and nondynamic parts, and takes the latter from normally a minimum CAS-CI wave function. ${ }^{47}$ However, in this study, we find that the minimal CAS-CI based on the Hartree-Fock orbitals does not provide the subtle effects required to obtain highly accurate results since the CAS-CI wave function itself is inaccurate. Consequently, much more effort is devoted to obtain a CAS with orbital optimization. After doing this, though, the TCCSD performs extremely accurately for this delicate and pathological example.

\section{ACKNOWLEDGMENTS}

This work was supported in part by U.S. Army Research Office under MURI (Contract No. C00005071-4). We also 
appreciate Professor Aoyagi and the staff at the computing and communications center, Kyushu University in Japan for allowing us to perform some calculations on the workstation they administer.

${ }^{1}$ P. J. Hay, T. H. Dunning, Jr., and W. A. Goddard III, J. Chem. Phys. 62, 3912 (1975)

${ }^{2}$ P. J. Hay and T. H. Dunning, Jr., J. Chem. Phys. 67, 2290 (1977).

${ }^{3}$ W. D. Laidig and H. F. Schaefer, J. Chem. Phys. 74, 3411 (1980).

${ }^{4}$ S. M. Adler-Golden, S. R. Langhoff, C. W. Bauschlicher, Jr., and G. D. Carney, J. Chem. Phys. 83, 255 (1985).

${ }^{5}$ T. Tanaka and Y. Morino, J. Mol. Spectrosc. 33, 538 (1970).

${ }^{6}$ A. Barbe, C. Secroun, and P. Jouve, J. Mol. Spectrosc. 49, 171 (1974).

${ }^{7}$ Y. Yamaguchi, M. J. Frisch, T. J. Lee, H. F. Schaefer, and J. S. Binkley, Theor. Chim. Acta 69, 337 (1986)

${ }^{8}$ T. J. Lee, W. D. Allen, and H. F. Schaefer III, J. Chem. Phys. 87, 7062 (1987).

${ }^{9}$ K. A. Peterson, R. C. Mayrhofer, E. L. Sibert, and R. C. Woods, J. Chem. Phys. 94, 414 (1991).

${ }^{10}$ P. Borowski, K. Andersson, P. A. Malmqvist, and B. O. Roos, J. Chem. Phys. 97, 5568 (1992).

${ }^{11}$ K. Andersson, P.-A. Malmqvist, B. O. Roos, A. J. Sadlej, and K. Wolinski, J. Phys. Chem. 94, 5483 (1990).

${ }^{12}$ K. Andersson, P.-A. Malmqvist, B. O. Roos, A. J. Sadlej, and K. Wolinski, J. Chem. Phys. 96, 1218 (1992).

${ }^{13}$ J. Olsen, B. O. Roos, P. Jorgensen, and H. J. A. Jensen, J. Chem. Phys. 89, 2185 (1988)

${ }^{14}$ J. Almlof and P. R. Taylor, J. Chem. Phys. 86, 4070 (1987).

${ }^{15}$ J. F. Stanton, W. N. Lipscomb, D. H. Magers, and R. J. Bartlett, J. Chem. Phys. 90, 1077 (1989).

${ }^{16}$ D. H. Magers, W. N. Lipscomb, R. J. Bartlett, and J. F. Stanton, J. Chem. Phys. 91, 1945 (1989).

${ }^{17}$ G. E. Scuseria, T. J. Lee, A. C. Scheiner, and H. F. Schaefer III, J. Chem. Phys. 90, 5635 (1989).

${ }^{18}$ T. J. Lee and G. E. Scuseria, J. Chem. Phys. 93, 489 (1990).

${ }^{19}$ J. D. Watts, J. F. Stanton, and R. J. Bartlett, Chem. Phys. Lett. 178, 471 (1991).

${ }^{20}$ R. J. Bartlett, Int. J. Mol. Sci. 3, 579 (2002).

${ }^{21}$ G. D. Purvis and R. J. Bartlett, J. Chem. Phys. 76, 1910 (1982).

${ }^{22}$ K. Raghavachari, G. W. Trucks, J. A. Pople, and E. Replogle, Chem. Phys. Lett. 158, 207 (1989).

${ }^{23}$ J. A. Pople, M. Head-Gordon, and K. Raghavachari, J. Chem. Phys. 87, 5968 (1987).

${ }^{24}$ K. Raghavachari, G. W. Trucks, J. A. Pople, and M. Head-Gordon, Chem. Phys. Lett. 157, 479 (1989).
${ }^{25}$ R. J. Bartlett, J. D. Watts, S. A. Kucharski, and J. Noga, Chem. Phys. Lett. 165, 513 (1990).

${ }^{26}$ T. H. Dunning, Jr., J. Chem. Phys. 53, 2823 (1970); S. Huzinaga, ibid. 42, 1293 (1965).

${ }^{27}$ J. D. Watts and R. J. Bartlett, J. Chem. Phys. 108, 2511 (1998).

${ }^{28}$ S. A. Kucharski and R. J. Bartlett, J. Chem. Phys. 110, 8233 (1999).

${ }^{29}$ R. A. Chiles and C. E. Dykstra, J. Chem. Phys. 74, 4544 (1981); L. Z. Stolarczyk and H. J. Monkhorst, Int. J. Quantum Chem., Quantum Chem. Symp. 18, 267 (1984); J. F. Stanton, J. Gauss, and R. J. Bartlett, J. Chem. Phys. 97, 5554 (1992); S. Larsson and V. H. Smith, Jr., Phys. Rev. 178, 137 (1969).

${ }^{30}$ N. C. Handy, J. A. Pople, M. Head-Gordon, K. Raghavachari, and G. W. Trucks, Chem. Phys. Lett. 164, 185 (1989); R. Kobayashi, R. D. Amos, and N. C. Handy, J. Chem. Phys. 100, 1375 (1994).

${ }^{31}$ J. Noga and R. J. Bartlett, J. Chem. Phys. 86, 7041 (1987).

${ }^{32}$ G. E. Scuseria and H. F. Schaefer III, Chem. Phys. Lett. 152, 382 (1988).

${ }^{33}$ T. H. Dunning, Jr., J. Chem. Phys. 90, 1007 (1989).

${ }^{34}$ S. A. Kucharski and R. J. Bartlett, J. Chem. Phys. 108, 9221 (1998).

${ }^{35}$ M. L. Leininger and H. F. Schaefer III, J. Chem. Phys. 107, 9059 (1997).

${ }^{36}$ P. Saxe, D. J. Fox, H. F. Schaefer, and N. C. Handy, J. Chem. Phys. 77, 5584 (1982); R. S. Grev and H. F. Schaefer, ibid. 96, 6850 (1992).

${ }^{37}$ D. Xie, H. Guo, and K. A. Peterson, J. Chem. Phys. 112, 8378 (2000).

${ }^{38}$ H.-J. Werner and P. J. Knowles, J. Chem. Phys. 89, 5803 (1988).

${ }^{39}$ P. J. Knowles and H.-J. Werner, Chem. Phys. Lett. 145, 514 (1988).

${ }^{40}$ S. R. Langhoff and E. R. Davidson, Int. J. Quantum Chem. 8, 61 (1974).

${ }^{41}$ I. Hubac, J. Pittner, and P. Carsky, J. Chem. Phys. 112, 8779 (2000).

${ }^{42}$ J. Pittner, P. Nachtigall, P. Carsky, J. Masik, and I. Hubac, J. Chem. Phys. 110, 10275 (1999).

${ }^{43}$ X. Li and J. Paldus, J. Chem. Phys. 110, 2844 (1999).

${ }^{44}$ X. Li and J. Paldus, J. Chem. Phys. 107, 6257 (1997); X. Li and J. Paldus, ibid. 108, 637 (1998).

${ }^{45}$ P. G. Szalay and R. J. Bartlett, Chem. Phys. Lett. 214, 481 (1993).

${ }^{46}$ K. W. Sattelmeyer, H. F. Schaefer III, and J. F. Stanton, Chem. Phys. Lett. 378, 42 (2003).

${ }^{47}$ K. Kowalski, J. Chem. Phys. 123, 014102 (2005).

${ }^{48}$ T. Kinoshita, O. Hino, and R. J. Bartlett, J. Chem. Phys. 123, 074106 (2005).

${ }^{49}$ N. Oliphant and L. Adamowicz, J. Chem. Phys. 95, 6645 (1991).

${ }^{50}$ M. W. Schmidt, K. K. Baldridge, J. A. Boatz et al., J. Comput. Chem. 14, 1347 (1993).

${ }^{51}$ T. H. Dunning, Jr., J. Chem. Phys. 55, 716 (1971).

${ }^{52}$ S. A. Kucharski, J. D. Watts, and R. J. Bartlett, Chem. Phys. Lett. 302, 295 (1999); M. Musial, S. A. Kucharski, and R. J. Bartlett, J. Mol. Struct.: THEOCHEM 547, 269 (2001).

${ }^{53}$ S. A. Kucharski, M. Kolaski, and R. J. Bartlett, J. Chem. Phys. 114, 692 (2001). 